\title{
¿Cómo se constituyen las experiencias de maltrato machista? La violencia simbólica y explícita basada en género como derrotero de juventudes universitarias. 1
}

\author{
How are sexist abuse experiences constituted? Symbolic and explicit \\ gender-based violence as a course for university youths.
}

\author{
Paula Flores-Aguilar ${ }^{*}$ \\ Claudia Contreras Contreras *
}

\section{Resumen}

El artículo describe factores que constituyen y contextualizan experiencias de violencia basada en género (VBG) vivenciadas por juventudes de la Universidad Austral de Chile. Los resultados expuestos obedecen a un análisis cualitativo de relatos orales y escritos, desarrollado con teoría fundamentada. Los hallazgos dieron cuenta que la presencia y encadenamiento de los siguientes elementos es fundamental en el cómo se vive la VBG, a saber: identidad de género, roles/estereotipos de género, figuras significativas, tramos de vida, contextos de VBG y aspectos psicológicos generales. Se concluyó que la VBG -simbólica y explícita- afecta las identidades de las juventudes desde su esencia, haciéndose patente a través de la endoculturación y el aprendizaje social. Dichas acciones tienen alcance longitudinal y se viven prioritariamente en contextos como: familia, amistades, escuela y redes sociales, involucrando principalmente a figuras como la madre, el profesor (figura masculina) y la pareja.

\footnotetext{
${ }^{1}$ Investigación financiada por la Agencia Nacional de Investigación y Desarrollo, ANID-CHILE - PCHA / BECA DOCTORADO NACIONAL 2014 - FOLIO 21130868. Investigación realizada en el marco del Programa de Investigación Asociativa de la Agencia Nacional de Investigación y Desarrollo, ANID-CHILE, a través del Proyecto Anillo: "CONVERGING HORIZONS: PRODUCTION, MEDIATION, RECEPTION AND EFFECTS OF REPRESENTATIONS OF MARGINALITY", PIA-ANID/ANILLOS SOC180045.

* Doctora en Ciencias Humanas, Mención Discurso y Cultura, Mg. en Comunicación y Periodista, Universidad Austral de Chile. Investigadora en Estudios de Género, Tecnologías de la Información y la Comunicación, Juventud e Infancia. Integrante del Centro Internacional de Estudios Frontera https://orcid.org/0000-0002-7223-002X Correo electrónico: florespaulamf@gmail.com

** Doctora en Psicología de la Educación, Máster en Psicología de la Educación, Psicóloga y Educadora de Párvulos. Académica del Instituto de Ciencias de la Educación, Facultad de Filosofía y Humanidades, Universidad Austral de Chile. Investigación orientada al análisis de incidentes críticos en la docencia y la socialización de género en educación escolar. https://orcid.org/0000-0002-7717-6835 Correo electrónico: claudia.contreras@uach.cl
} 
Palabras clave: violencia (meta)simbólica basada en género, juventudes, experiencias, factores influyentes, identidad de género

\begin{abstract}
The article describes factors that constitute and contextualize experiences of genderbasedviolence (GBV) lived by young people of the Universidad Austral de Chile. The results are based on a qualitative analysis of oral and written accounts, developed from grounded theory. The findings showed that the presence and chaining of the following elements is fundamental in how GBV is experienced, namely: gender identity, gender roles/stereotypes, significant figures, life spans, contexts of GBV and general psychological aspects. It was concluded that GBV symbolic and explicit- affects the identities of young people from their essence, becoming evident through endoculturation and social learning. Such aggressions have a longitudinal scope and are experienced primarily in contexts such as: family, friends, school and social networks, mainly involving figures such as the mother, the teacher and the romantic partner.
\end{abstract}

Keywords: (meta) symbolic gender-based violence, youth, experiences, influencing factors, gender identity

Fecha de recepción: Mayo 2021

Fecha de aprobación: Diciembre 2021

\title{
1. Introducción
}

Los tiempos que corren dan cuenta de una agitada realidad local e internacional que, como fruto del despertar social experimentado en el último tiempo, continuamente busca mecanismos a través de los cuales transformarse y evolucionar. Desde la cuarta ola feminista, Chile y Latinoamérica han sido testigos de una seguidilla de organizaciones y levantamientos que, persiguiendo la equidad de género, se han erigido como síntomas de una sociedad cansada de la hegemonía masculina.

Dicho hartazgo obedece a esquemas materiales y simbólicos inmemoriales que, en base a la díada femenino/masculino, han dado forma a la cultura, donde el hombre se ha configurado como figura de poder omnipotente y cuya propiedad sobre el espacio público ha posibilitado la producción y reproducción de lógicas de dominación en todas las esferas socioculturales. Las mujeres, en cambio -históricamente relegadas al espacio privado y a los roles de cuidado-, han acostumbrado a construirse desde la alteridad. A partir de este contexto, la violencia basada en género se ha tornado un fenómeno omnipresente, frecuente y naturalizado, toda vez que encuentra justificación en los sistemas de creencias fundantes de la cultura occidental. 
Así, desde los primeros feminismos -cuyo auge se alcanzó durante el siglo XVIII- la problemática de la agresión contra la mujer ha sido foco de atención y necesaria reflexión. Si bien en la historia reciente los mandatos sociopolíticos han debido ceder a las exigencias por la equidad de derechos y deberes, las manifestaciones de violencia no han cesado, sino que inclusive- han aumentado. En este sentido las cifras anuales de violaciones, femicidios, humillaciones y tratos vejatorios hacia las mujeres dan cuenta de un escenario que conserva en su esencia imaginarios violentos (Ruiz-Pérez y Pastor-Moreno, 2020).

Desde la investigación académica, diferentes estudios nacionales e internacionales se han orientado al estudio del fenómeno en áreas específicas como el ambiente laboral (Díaz et al., 2017; Acevedo y Borges, 2009), la realidad de mujeres con ascendencia étnica (Zapata et al., 2012), contextos de enseñanza-aprendizaje (Bernal, 2005; Madrid, 2006), relaciones de pareja (Gómez et al., 2015; Axt et al., 2019; Rodríguez-Castro y Alonso-Ruido, 2015), en la vía pública (Arancibia y González, 2017) en el sistema penal e institucional (Bodelón, 2014), entre otros. Además, en el contexto latinoamericano existen investigaciones que abordan la violencia de género en la juventud desde reflexiones teórico-conceptuales (Zurbano Berenguer et al., 2015), dimensiones perceptivas del fenómeno (De Miguel, 2005), desde experiencias virtuales con formatos -más o menos- explícitos (Donoso Vázquez et al., 2018; Alegría y Rodríguez, 2015) y en el ambiente universitario (González y Carapias, 2015; Martín, 2012; Zamudio et al., 2017); sin embargo, persiste el desafío de una aproximación holística e integradora, que comprenda y aborde dimensiones fundamentales como los esquemas simbólicos que sustentan la cultura machista y las causas que subyacen a determinados posicionamientos y significaciones juveniles respecto a distintas formas de violencia basada en género.

En relación con lo antes expuesto, el objetivo del presente artículo es describir aquellos factores que influyen en cómo los y las estudiantes de la Universidad Austral de Chile (UACh) significan la violencia de género desde sus experiencias de vida.

\section{Perspectiva teórica}

\subsection{Patriarcado: cimientos y contracorrientes}

Para situarnos en el fenómeno de la violencia de género, debemos entender al patriarcado como un sistema político, social, económico, cultural, sexual y religioso que establece (explícita e implícitamente) pautas de comportamiento y relación basadas en la inequidad, en la jerarquía de lo masculino por sobre lo femenino, en el desmedro, debilitamiento, cosificación y control de todo aquello que provenga o se asocie a las mujeres y en el ensalzamiento, sentimiento de propiedad y poder que per sé se otorga a los hombres ( $\mathrm{y}$ a lo masculino en general), quien, desde la cima, goza de un sinnúmero de ventajas simbólicas y materiales en todo aspecto de la socialización. 
Este marco social, amparado y justificado en características biológicas, históricamente ha derivado en la construcción binaria del género (Segato, 2016; de Beauvoir, 1999, Butler, 2007) y caracteriza a nuestra sociedad occidental y latinoamericana, trayendo consigo consecuencias como la excesiva estereotipación, la identidad de género rigidizada, los mitos del amor romántico y la violencia machista simbólica y explícita, entre muchas otras.

En este entramado, la familia heteronormada, entendida como sistema nuclear básico del sistema patriarcal, se transforma en el elemento básico que -manteniendo lógicas de jerarquía masculina en roles y estereotipos, así como en el origen y desarrollo de sus relaciones sexo-afectivas- consolida y perpetúa al machismo desde su esencia (de Beauvoir, 1999; Facio, 1999; Sendón de León, 2003; Fontenla, 2008). A partir de la intimidad y cotidianeidad del sistema familiar, las lógicas de inequidad se moldean y proyectan hacia las esferas sociales públicas, mismas que desde todos sus frentes, actúan sinérgicamente para sostener al sistema de la fratria.

Así, la familia y las relaciones de poder que ahí se desarrollan son una evidencia -a pequeña escala- de las lógicas que rigen a nuestros esquemas socioculturales. En ambos espacios, son las figuras masculinizadas quienes proveen de recursos materiales y simbólicos, concentrando el poder (económico, político e ideológico), estableciendo mecanismos de control más o menos explícitos, dirigidos a mantener la subordinación -paradójicamente cómplice y hasta agradecida- de los grupos inferiores marginados (mujeres, niños/as, pobres).

De este modo el patriarcado se presenta como un sistema fractal capaz de abarcar todos los campos sociales y afectar la individualidad de hombres, mujeres y otres. No obstante, se hace preciso detenernos en el carácter artificial de dicho sistema, que -aunque naturalizadoobedece a una construcción basada en lógicas colonialistas del género, convirtiéndose en una barbarie que atenta contra la paz social (Sendón de León, 2019).

\subsection{El artificio del género binario y sus consecuencias}

El género aún es comúnmente asociado a un orden natural de las cosas (Bourdieu, 2000), una especie de esquema que desde siempre ha categorizado a hombres y mujeres con determinados roles, características, estereotipos, derechos y deberes. Sin embargo, y aludiendo a debates cada vez más frecuentes, la categoría del género encarna modos de ser y estar que, valiéndose de características biológicas del ser humano y su consecuente división sexual del trabajo (de Beauvoir, 1999; Varela, 2017), han sido construidos por la sociedad y, por tanto, no son más que imaginarios artificiales.

Sin embargo, dicha artificialidad no resta a su determinismo, pues estableciéndose como un "conjunto de características y comportamientos, roles, funciones y valoraciones impuestas 
dicotómicamente a cada sexo a través de procesos de socialización, mantenidos y reforzados por la ideología e instituciones patriarcales" (Facio, 1999, p. 13), su efectividad llega a decretar la identidad y experiencia de vida de toda persona, adaptándose a distintas culturas, realidades y escenarios interseccionales.

Desde el plano discursivo, los estudios de Judith Butler (2007) reconocen al género como una construcción dada a partir de la repetición de actos estilizados, que logran naturalizar los comportamientos e identidades asociados a las prácticas reguladoras que se imponen sobre la díada femenino/masculino, estableciendo categorías inteligibles que permiten la inclusión social. Se trata de una categoría, que -aunque forzosamente unida a la idea del sexo biológicoencuentra su derrotero en la producción y reproducción de mecanismos inequitativos, solapados en nuestra cultura y que, atravesados por dimensiones raciales, económicas, políticas, religiosas y de clase (Facio, 1999), hacen de cada identidad algo único, variable y distinguido dentro del universo de la subordinación.

En otra perspectiva, pero conservando la esencia argumental, autores como Gerard Coll-Planas (2013) y Rita Segato (2016) sitúan al género desde su naturaleza en tanto categoría que nos permite entender y leer la sociocultura de la cual somos parte y que obedece a la conveniencia de los grupos de poder masculinos, los que en su misión de mandatar la construcción del binomio cuerpo-género, limitarían a los sujetos a una realidad totalizadora, en la cual es "(...) el género el que crea el sexo. Por un lado, porque el género es lo que da significado a nuestros cuerpos, nos dice cómo debemos leerlos. Por otro lado, porque los ideales normativos de género nos llevan a transformar nuestros cuerpos." (Coll-Planas, 2013: 27-28). A tal respecto, el género representaría una configuración histórica elemental de la violencia, toda vez que fabrica y estampa los sellos de las relaciones asimétricas, donde el poder es expropiado por una de las partes.

Podemos, entonces, ser conscientes de algunas certezas: la categoría del género no es natural (pero sí naturalizada), obedece a una construcción activa por parte del sujeto (cuerpo sexuado) a partir de las dimensiones inteligibles que la cultura le ofrece; varía en relación a su situación histórica y sociocultural; es inherente a la identidad de las personas, hecho que las reglamenta, moldea y (trans)forma; no es una condición estable, sino sujeta a tantos cambios como la experiencia de vida lo requiera; busca mantener el orden social patriarcal a través de la heterosexualidad normada y mandata lo (in)adecuado en el marco de las relaciones interhumanas, generando marginación hacia los grupos dominados y subversivos.

\subsection{Juventudes e identidades atravesadas por la violencia basada en género}

La construcción identitaria abarca dimensiones trascendentales para el modo en que, paralela y posteriormente, las juventudes experimentarán la violencia de género a lo largo de la 
vida. Por ello resulta necesario conocer algunos factores esenciales implicados en dicho proceso, pues sólo desde ahí se puede proyectar una resignificación del tránsito mencionado.

Entendiendo a la identidad de género como una construcción personal que involucra el reconocimiento de la singularidad, la unicidad y la exclusividad, al tiempo que significa una importante construcción social, referida a la pertenencia (Rocha, 2009) y capaz de formarse gracias a la integración de los diversos aspectos históricos y culturales con la individualidad del sujeto (Parsons, 1968), debemos entender a éste como un desarrollo situado históricamente en el macrosistema patriarcal.

Actualmente resultaría prácticamente impensado crear una identidad de género fuera de las normas machistas, atendiendo a que infantes, niños/as y jóvenes se adhieren a los roles/estereotipos de género de forma acrítica y que desafiar a la norma, significaría poner en riesgo el sentido de pertenencia social.

En tal sentido, se puede afirmar que los estereotipos de género, entre otros, constituyen la base de la construcción de la identidad de género (Lagarde, 2004), orientando y guiando acciones, pensamientos, comportamientos y representaciones de la realidad expresadas por las personas (Jiménez, 2005 en Colás y Villaciervos, 2007). A partir de esta encrucijada se generaría una estrecha reciprocidad entre estereotipos, roles e identidad de género, tríada que explicaría la (re)producción de pensamientos y acciones machistas, en planos individuales y colectivos.

La mirada recién expuesta, se plantea como la antesala que configura la existencia femenina desde características cuidadoras, pasivas, (hiper)sexualizadas y sumisas (de Beauvoir, 1999; Facio, 1999; Varela, 2017). En la otra vereda la masculinidad toma forma gracias a imaginarios de independencia, virilidad, inteligencia, lógica, libertad y control -tanto de su entorno como de las mujeres que lo habitan- (Colás y Villaciervos, 2007; Varela, 2017; Marañón, 2018).

El proceso identitario que deviene a partir del escenario descrito, y que resulta especialmente crítico durante la adolescencia y la juventud (López, 2016), obedece a un juego complejo acunado por el sistema de género, mismo que -intervenido por múltiples referentes culturales- determinaría cómo se lleva a cabo la construcción del "ser hombre" o "ser mujer".

Ahora bien, a los estereotipos descritos se suman variables fundamentales para la construcción del "self', como la intervención e influencia de figuras claves, elementos cognitivos y motivacionales (asociados al capital cultural y expectativas de pertenencia social) y las experiencias de violencia experimentadas por razón de género. 
Los procesos que abrazan los elementos antes descritos, y que explicarían el modo en que los sujetos desde su infancia interiorizan y aprehenden comportamientos ligados al género, son la endoculturación y el aprendizaje social.

Por endoculturación, entendemos todas aquellas vivencias basadas en la comunicación y otros simbolismos, gracias a las cuales los sujetos van adquiriendo y haciendo propias ciertas características culturales, sin ser conscientes de ello (Díaz - Guerrero, 1972 en Rocha, 2009). Este fenómeno ocurriría especialmente durante la infancia y la primera parte de la adolescencia, donde -generalmente- aún no se han desarrollado capacidades para hacer frente al bombardeo estereotipado de su entorno, período donde además hay una alta sensibilidad en cuanto a la definición personal y donde se tiende a buscar la aprobación del entorno. En este contexto los comportamientos y características observados en las figuras de referencia se vuelven realidades inequívocas, transformándose en expectativas de un ideal identitario.

En la misma línea, la teoría del aprendizaje social, desarrollada por Bandura (1977) afirma que las personas aprenden a ser masculinos o femeninos a través de la comunicación y la observación de su entorno (padre, madre, amigos/as, medios de comunicación, entre otros). El proceso de aprendizaje, dado a partir de dichas interacciones, permite entonces que los/as niños/as interioricen y desarrollen "los comportamientos y características que son asociados a la masculinidad y a la feminidad y conforme crecen, continúan imitando aquellas conductas que dan pauta a una comunicación e intercambio efectivo con los otros" (Rocha, 2009, p.254).

A este respecto, lejos de entender la juventud como una categoría inequívoca, estable y universal, la significaremos como un estado donde influyen y confluyen elementos como la raza, el origen, nivel socioeconómico, género, contexto religioso, político y sociocultural (Alpízar y Bernal, 2013; Duarte, 2018), por lo que debiese ser comprendido desde una mirada dialogante, flexible, pluralista y alejada del adultocentrismo. Así, la condición de "ser joven", en el contexto de este estudio y situada en una sociedad tecnologizada (Tortajada, 2018; Ruiz, 2012), se observa como una oportunidad, toda vez que permite pensar en sujetos autónomos, actores políticos con plasticidad en el desarrollo de sus identidades, con capacidad de transformar sus entornos y también de adoptar -o en algunos casos reformular- patrones culturales tradicionales (Hein y Cárdenas, 2009 en Duarte, 2018).

Así es como la identidad de género en las juventudes actuales se presenta como un tránsito multifactorial, intervenido por distintos procesos, figuras y elementos que conservan mucho de la tradición machista, pero que también ofrecen oportunidades para la redefinición de imaginarios culturales que sostienen a los géneros y los modos de relación entre sí. 


\subsection{Violencia basada en género desde la teoría y la praxis}

La violencia, expresada a través de innumerables canales reales y simbólicos, surge como respuesta a las tensiones generadas por la asimetría de poder entre géneros en el marco de una sociedad androcéntrica, que al tiempo determinan nuestros imaginarios sociales. Podemos ver síntomas del patriarcado en nuestras infancias limitadas (y muchas veces olvidadas) por la imposición -adultocéntrica- del deber ser, en adolescencias frustrantes y cansadas de la lucha por encajar y pertenecer; en una vida adulta plagada de normas y estigmas; en el mercado que, validando parámetros materiales de felicidad, regula nuestros comportamientos y nos transforma en objetos de consumo (Segato, 2016; Marañón, 2018) en la educación que perpetúa la desigualdad; en la justicia que violenta a la mujer con su desidia e invisibilización; en el Estado paternalista que concentra el poder en lo masculino, que regula en pos de una sociedad heteronormada y que decide sobre la vida femenina con la escasa -o nulapresencia de mujeres; en la economía que acude a los cuerpos femeninos con el principal fin de exhibirlos en tanto objetos de placer, susceptibles de ser adueñados por una otredad masculina.

De lo antes dicho, la violencia de género encontraría su asidero en las construcciones de las que hasta ahora hemos reflexionado. Se trataría de un tejido que, atravesando lo individual, público y privado, contaminaría a las figuras masculinas mediante la imposición de expectativas asociadas al poder y control, con los consecuentes sentimientos de validación social o de frustración agresiva, según sea el caso (Ramírez Rodríguez et al., 2009; Marañón, 2018).

Según John Galtung la violencia derivada de dicho fenómeno, experimentada mayormente en los contextos de familia y pareja (Ramírez Rodríguez et al., 2009), se podría categorizar como: a) directa: expresada de manera explícita o fácilmente reconocible; b) estructural: manifestaciones machistas que marginan, excluyen, explotan y dominan al sujeto femenino, y c) cultural: apelando a los sistemas de creencias patriarcales que atraviesan la sociedad y que están presentes en "razonamientos, actitudes e ideas que justifican, legitiman y promueven la violencia en sus formas directa o estructural (Galtung, 1995 en Espinar Ruiz y Mateo Pérez, 2007, p. 191).

En el mismo sentido, las expresiones de violencia por razón de género podrían ser de origen simbólico o bien, metasimbólico. La primera categoría se reconoce en las teorizaciones de Pierre Bourdieu, quien caracteriza a la violencia como aquella que:

“(...) se define en y por una relación determinada entre los que ejercen el poder y los que los sufren, es decir, en la estructura misma del campo donde se produce y se reproduce la creencia. (...) El poder simbólico, poder subordinado, es una forma transformada -es decir, irreconocible, transfigurada y legitimada-, de las otras formas de poder (...)" (Bourdieu, 2000, p. 4-5). 
Entonces, desde la lógica de la subordinación, la violencia simbólica se encarnaría en todas aquellas expresiones, lógicas, estructuras y sistemas que, valiéndose de un capital simbólico, político, económico y social, mantendrían el orden hegemónico-masculino contando con la venia de los grupos dominados.

Ahora bien, más allá de la violencia simbólica de género, encontramos aquella violencia cuyo origen es metasimbólico, toda vez que dicha agresión encuentra su asidero en imaginarios simbólicos, al tiempo que lo transgreden, representándose en actos directos y explícitos, que generalmente son los más evidentes y -por tanto- fáciles de reconocer y condenar. Se trata de malos tratos evidentes (generalmente físicos, psicológicos y/o sexuales) que encierran en sí lo explícito e implícito de la violencia machista, y que representan la teoría y el pragmatismo del modelo machista. En el caso de una violación, por ejemplo, la violencia metasimbólica se ve expresada en el acto físico de forzar y someter a una mujer a determinados actos sexuales, mientras que -en el mismo caso- la violencia simbólica estaría representada en la relación asimétrica de poder que se ha construido entre hombres y mujeres, estructura relacional que históricamente le ha otorgado a lo masculino el derecho de satisfacer sus necesidades y expectativas, valiéndose de la cosificación, hipersexualización, control y apropiación del cuerpo femenino.

En el tránsito hacia una sociedad moderna, la violencia metasimbólica -o directa- crea cada vez mayor rechazo en la sociedad que la observa, configurado un escenario donde los maltratos simbólicos alcanzan especial relevancia para el mantenimiento del statu quo. Hablamos de expresiones reproductoras de habitus hegemónico-masculinos, intrincados en las estructuras, instituciones, cuerpos y discursos de las sociedades que lo padecen y que podemos palpar -por ejemplo- en el hecho de que el $98 \%$ de los bienes de la tierra esté en poder de hombres, o en que las 225 personas más ricas del mundo (varones en su totalidad) acumulen el mismo capital que los 2.500 millones más pobres, de los cuales el $80 \%$ son mujeres (Segato, 2016).

Así, el juego perverso de lo simbólico se hace patente desde lo macro a lo micro, a través de fenómenos como el coeficiente simbólico negativo o, en palabras de Sendón de León (2003), coeficiente simbólico femenino, concepto utilizado para dar cuenta de la connotación negativa, pre y perjuiciosa que realiza la sociedad a todo aquello que sea femenino, o que se le relacione.

En el mismo sentido, surgen las nuevas misoginias (Varela, 2017) y postmachismos (Lorente, 2009), fenómenos que dejan ver cómo los abusos machistas de siempre evolucionan y adoptan nuevas formas (generalmente simbólicas) de denigración hacia la mujer, manteniendo el modelo de la supremacía masculina bajo caretas que -quedándose sólo en el discurso- aparentan equidad en una "cultura del simulacro" (Varela, 2017). 
Estamos situados entonces en una sociocultura que, a nivel privado, público, estatal, estructural e individual y por acción u omisión, permite, valida y legitima la existencia y proliferación de los estereotipos, la inequidad y -por consecuencia- la violencia contra la mujer.

\section{Metodología}

Este artículo forma parte de un proceso de investigación doctoral, el cual buscó comprender las expresiones de violencia basada en género protagonizadas por los/as jóvenes de la Universidad Austral de Chile (UACh), a partir del análisis crítico de sus relatos orales y escritos. La investigación se realizó a partir de metodología sociocrítica con enfoque combinado, específicamente desde el método mixto secuencial con estatus dominante (cuantitativo - CUALITATIVO) (Hernández, Fernández y Baptista, 2003; Johnson y Onwuegbuzie, 2004 y Onwuegbuzie y Leech, 2006 en Pereira, 2011).

Los resultados que aquí se exponen corresponden a la fase cualitativa del estudio, desarrollada a partir de los principios de la teoría fundamentada (Strauss y Corbin, 2002) y que incorporó algunos elementos de la metodología comunicativa crítica (Gómez et al., 2016). La elección descrita pretende relevar la comprensión de la realidad como una construcción intersubjetiva, dada la significación común de realidades espaciotemporales realizada por las juventudes estudiadas, a fin de lograr plasmar aquello que ven como su realidad social (BonillaGarcía y López-Suárez, 2016). De este modo, se buscó dar valor las perspectivas e interpretaciones que los/as jóvenes hacen de sí mismos/as y de sus acciones sociales, y además aminorar la brecha jerárquica que tradicionalmente caracteriza la relación entre investigadora y sujetos/as de estudio.

Durante el trabajo de campo se realizaron tres grupos de discusión, conformados por un total de doce estudiantes de la UACh de las áreas de pre y posgrado. Dos de los grupos estuvieron compuestos únicamente por personas identificadas con el género femenino (8 mujeres en total), mientras que el tercer grupo contó con la participación de ambos géneros ( 2 mujeres y 2 hombres). Si bien se buscó intencionar la participación equitativa de mujeres y hombres, la marcada presencia minoritaria de estos últimos da cuenta de una posible falta de interés por el fenómeno de estudio.

Las sesiones se dividieron en dos momentos: al inicio cada participante redactó un testimonio voluntario sobre alguna experiencia de violencia contra la mujer que haya sido significativa para ellos/as; en una segunda instancia, se abrió el espacio para la conversación guiada. 
Cabe destacar que, en concordancia con los principios de la metodología comunicativa crítica (Gómez et al., 2016), las temáticas a abordar en las sesiones fueron compartidas y retroalimentadas por los/as jóvenes previo a la aplicación de los grupos de discusión.

El análisis de la información se realizó con el apoyo del software ATLAS.ti 8 y se basó en el paradigma de la teoría fundamentada, incluyendo aspectos asociados al interaccionismo simbólico. Dicho abordaje da cabida a una mayor flexibilidad metodológica en la generación de conocimiento, toda vez que valida la complementación entre categorías deductivas e inductivas de análisis y rescata el conocimiento científico previo, beneficiando la integridad y profundidad del conocimiento alcanzado (Bonilla y López, 2016; San Martin Cantero, 2014).

A tal efecto, el estudio de los datos consistió en: a) análisis basado en conceptualizaciones teóricas previas, b) codificación abierta (identificación y establecimiento de códigos iniciales); c) codificación axial (depuración de códigos y establecimiento de relaciones para la conformación de categorías o grupos de códigos) y d) codificación selectiva (establecimiento de categorías centrales y relaciones entre categorías para la generación de teoría emergente acerca de la realidad estudiada). Además, el proceso descrito permitió alcanzar tres niveles de análisis en torno al objetivo propuesto, a saber: a) frecuencia de códigos, b) co-ocurrencia de códigos y c) redes semánticas.

El estudio constó de fases iterativas, durante las cuales se revisaron, depuraron y precisaron los sistemas de codificación. El resultado final de análisis arrojó un total de 253 códigos, categorizados en 20 categorías o grupos de códigos.

\section{Resultados}

\subsection{Descripción de factores influyentes en el modo de vivir la violencia machista}

El cumplimiento del objetivo planteado, que buscaba "describir los factores que influyen en cómo los y las estudiantes de la UACh experimentan la violencia de género y los contextos específicos en los cuales se ejerce", requirió de un abordaje complejo, debido a los múltiples componentes que se ven implicados en dicho fenómeno.

En relación con lo anterior, es necesario destacar que la categoría "identidad de género" alcanzó una notoria relevancia para el entendimiento del escenario estudiado, puesto que se observó que la postura adoptada frente a la violencia machista y/o la significación que realiza respecto a la misma, estaría principalmente determinada por la relación entre identidad y el resto de los conceptos fundamentales mencionados previamente. A tal efecto, la dimensión identitaria se plantea como hilo conductor de los resultados que -atendiendo al nivel de análisis de co-ocurrencia de códigos- se exhiben a continuación. 


\subsection{Identidad de género}

Al analizar los relatos de los/as jóvenes se pudo identificar que la violencia simbólica basada en género -representada en diversas experiencias a lo largo de sus vidas- resultan determinantes en la construcción de sus identidades (masculino, femenino y no binario).

Si bien este fenómeno se presenta para ambos géneros, en el caso de las mujeres la recurrencia de dicha categoría se vuelve trascendental, develando que existe una relación indisoluble entre este tipo de agresión invisibilizada y el modo en que las protagonistas (re)construyen su autopercepción en distintos momentos de sus historias de vida. La violencia de género moldea sus identidades y éstas, a su vez, determinan sus experiencias desde lo femenino/masculino.

\subsection{Roles y estereotipos de género}

En el mismo sentido, a la luz de los datos se pudo afirmar que la imposición de roles y estereotipos de género -propios del machismo y de la heteronormatividad- tienen una influencia trascendental en la construcción y expresión de las identidades de género de los/as jóvenes. Esto, sumado a los ideales sobre el amor romántico, evidenciaron que resulta prácticamente imposible divorciarse de los mandatos culturales machistas, pues prevalece la importancia de la familia tradicional para la continuidad del patriarcado y para una adecuada inserción en las normas sociales.

De este modo, se dificultaría abrir espacios para reconocer y expresar otras identidades de género, pues aún en los relatos asociados a lo queer (o no binario), las autopercepciones juveniles se construyeron incorporando una mixtura de rasgos femeninos y masculinos tradicionales.

Al profundizar sobre los roles y estereotipos asignados a hombres y mujeres, se observó que éstos, en su mayoría, obedecen al orden patriarcal. Lo masculino aún se perfila desde conceptos relacionados con la actividad y el espacio público, como el rol protector, virilidad, racionalidad, control, impulsividad, etc., mientras que lo femenino se construye desde el espacio privado y en un rol principalmente pasivo, atendiendo a aspectos como emocionalidad, recato, erotización para un tercero, sumisión, rol doméstico, rol de cuidadora, entre otros.

Como excepción a lo anterior, tres dimensiones se presentaron como disruptoras de la norma: el empoderamiento femenino, su consecuente sincretismo de género y la infantilización del hombre en el contexto de pareja. 
En este sentido, el empoderamiento de la mujer se suma como un rasgo moderno que acarrea consigo ciertas contradicciones respecto al rol asignado a lo femenino. Se trata del perfil paralelo que deben cumplir las mujeres (tradicional y moderno simultáneamente) quienes, junto con continuar cumpliendo el rol de cuidadoras, deben ahora desarrollarse e insertarse en el mercado laboral y el espacio público.

Del mismo modo, en los relatos analizados los estereotipos ligados al hombre hacen recordar al mitológico "complejo de Edipo": por un lado, se presenta la figura masculina que actúa de manera viril, con actitudes basadas en el control y la protección de sus círculos sociales privados (familia, pareja), a la vez que -en el contexto de una relación sentimental estable- se perfila bajo la figura de un niño, quien debe ser "atendido", "cuidado" y en algunos casos "mimado", como un hijo más.

\subsection{Aspectos psicológicos generales}

Al abordar los aspectos psicológicos presentes en los procesos de descubrimiento y formación de identidad relatados por los/as participantes, se pudo observar que fenómenos como la endoculturación (Rocha, 2009) y el aprendizaje social (Bandura, 1977; Bandura y Walters, 1963) son determinantes, entendiendo estos procesos como la aprehensión de patrones culturales a través de la interacción con el entorno, especialmente con figuras significativas de su entorno más próximo.

Se observó que la interiorización de esquemas sociales basados en lógicas machistas despierta en los/as jóvenes la necesidad de actuar en función del reconocimiento social y del sentimiento de pertenencia, situaciones que además generarían una autoestima positiva y reafirmarían la identidad de género. Por otra parte, evitar situaciones o actitudes que puedan desembocar en marginación y/o autoestima negativa e incluso recurrir a la alteridad defensiva (en el caso femenino), se vuelve también un mecanismo trascendente que guiaría la construcción del yo y mantendría regulados los comportamientos intra e interpersonales.

Se pudo advertir que la mayor parte de las experiencias relacionadas con la construcción de la autoestima y autopercepción tuvieron como base la niñez, donde se comenzaron a asentar las primeras sensaciones de inseguridad y frustración, a causa de las expectativas ligadas a la apariencia y a estereotipos propios de lo femenino. La asimilación de patrones culturales se daría principalmente gracias a la interacción con figuras clave, representadas principalmente por la madre, por uno o más profesores (masculinos) y, entrada la etapa de la adolescencia, por la pareja.

Otro espacio que se presentó como trascendente para la formación identitaria de los/as jóvenes, fue el de la escuela formal. Las experiencias relatadas que conectan a profesores de 
género masculino con episodios de violencia simbólica y contenido cargado de machismos propios del "currículum oculto", toman relevancia en épocas como la niñez y adolescencia, donde los/as participantes están especialmente vulnerables a la influencia de modelos y conductas externas, más aún cuando son ejercidas por una figura de autoridad y de cuidado.

\subsection{Tramos de vida y figuras clave}

Al analizar la presencia de figuras clave durante la formación identitaria de los/as jóvenes, se pudo identificar que -sobre todo en la niñez- la madre (o cuidadora) y el profesor (en masculino) ejercieron una fuerte influencia. Participantes los diversos géneros relataron situaciones recurrentes -vinculadas a violencia simbólica- donde el docente, de una u otra manera, marcó sus identidades de manera negativa, con marcada tendencia a la mantención del estereotipo. Más tarde, entrada la etapa de la adolescencia y juventud, sólo en el caso femenino la pareja alcanzó un rol relevante en cuanto a la autopercepción.

En tal sentido, los datos dieron cuenta que tanto la niñez, como la adolescencia y la juventud son épocas de vida donde recurrentemente intervienen factores que (re)definen la identidad de los/as participantes. En el caso del género no binario, el análisis advirtió que ciertas experiencias propias de la juventud fueron definitorias para el reconocimiento de dicha individualidad, hecho que sin duda se torna necesario de abordar en otro estudio.

El panorama antes expuesto da cuenta que las influencias sobre la identidad de género, ya sea bajo la figura de la madre, el docente (encarnado únicamente por figuras masculinas) o la pareja- se presentan a lo largo de todas las etapas de vida, variando en la frecuencia e intensidad de sus intervenciones, por lo que dicha autopercepción estaría sujeta a una permanente transformación.

\subsection{Contextos específicos de violencia en la construcción de identidad de género}

Dentro de los contextos en los que los/as jóvenes declararon haber vivido experiencias significativas para su identidad de género, se observó que la interacción experimentada en redes sociales, y en otros espacios privados y públicos fueron relevantes, específicamente aquellos ambientes que atañen al grupo familiar, a los/as amigos/as y a uno o más ambientes propios del sistema educativo formal. También se pudo ver que las situaciones violentas, vividas por las mujeres en la locomoción pública, tuvieron algún grado de influencia en el proceso antes mencionado. 


\subsubsection{Redes sociales}

Al advertir que el contexto de interacciones digitales fue uno de los que presentó mayor frecuencia de manifestaciones violentas implicadas en la construcción de la identidad, se decidió profundizar en su análisis.

En esta línea, se observó que los factores más influyentes de la red se dan principalmente en la etapa de la adolescencia, y dicen relación con la necesidad de validación y reconocimiento social, perseguidas a través de la exposición voluntaria. Se observó que la práctica de publicar fotografías del cuerpo femenino, así como de la vida privada, se vuelve un acto frecuente en la búsqueda de "likes", reafirmando con ello la naturalidad con la que se asume la cosificación femenina (Tortajada, 2018; Ruiz, 2012). También se vuelve significativa la presencia de comportamientos asociados al control en relaciones sentimentales, lo que, junto a otras características propias de una "relación tóxica", influirían en cómo las jóvenes viven y significan su identidad.

\subsection{Expectativas, cuerpo e identidad}

Respecto a la dimensión del análisis donde surgieron códigos espontáneos, se observó que en la construcción identitaria de las mujeres cobran especial importancia categorías vinculadas a las expectativas (auto)impuestas sobre la apariencia física, la vergüenza a sociada al propio cuerpo, la presión social del "deber ser" femenino. Así también, el sentimiento de vulnerabilidad y los mensajes transmitidos por los medios de comunicación han impactado de manera notoria la identidad de las participantes.

Otro aspecto observado como inherente al proceso de auto percepción fue el rol maternal, pues, en palabras de los/as jóvenes, la falta de éste desafiaría a las heteronormas, arriesgando algún tipo de castigo social. De este modo la validación social parece centrarse en el cuerpo, que se convierte en vehículo de autoestima y seguridad en tanto alcanza parámetros de erotización, belleza y fertilidad/gestación, propios de la sociedad machista.

En el caso de los varones, los participantes declararon ver influenciada su identidad de género por la necesidad de validación, especialmente aquella proveniente de las redes de amistad, mismas que intervienen significativamente en la subjetividad masculina. También se advirtieron como categorías influyentes el refuerzo ideológico dado por expresiones y conductas machistas -como chistes cargados de violencia simbólica- y el miedo al rechazo social, en caso de no ser condescendiente con los estereotipos y roles masculinos.

En cuanto a aquellos/as participantes que se refirieron a identidades de género no binarias, surgieron como necesidades esenciales la visibilización de la diversidad de géneros y 
la lucha de dichas disidencias por alcanzar respeto. Además, en la búsqueda de identidad, el apoyo y contención prestada por las amistades se volvió un factor crucial para alcanzar sentido de pertenencia, en caso de que éste no fuera encontrado en el seno familiar.

A modo genérico, los relatos dieron cuenta de la importancia que alcanzan ciertos mecanismos de resistencia frente a las normas patriarcales, entre los que destacaron la coeducación, el acceso al conocimiento sobre nuevas formas de construir el género y la producción de contenidos audiovisuales infantiles menos estereotipados y más inclusivos.

\subsection{Elementos de la violencia machista en perspectiva}

Desde un panorama holístico se puede señalar que la significación de la violencia de género simbólica y metasimbólica que realizan los/as jóvenes está determinada por la presencia e interacción de seis factores fundamentales; a saber, identidad de género, contextos de las experiencias de agresión machista, figuras clave, roles y estereotipos de género, tramo de vida y aspectos psicológicos generales, cada uno de los cuales comprende de categorías específicas y de características particulares.

De este modo, el escenario se configura a partir de la conjugación compleja de elementos, que a lo largo de la historia de vida aparecen con distintos niveles de fuerza, aportando a la sostenida y recurrente (re)formación de la identidad de género, dimensión que se plantea como eje ideológico desde el cual se entiende y experimenta la violencia machista.

\section{Conclusiones}

Dentro de aquellos elementos que inciden críticamente en cómo los/as jóvenes viven la violencia de género, surgieron seis dimensiones principales, las cuales -dando cuenta de una constante simbiosis y retroalimentación- lograron responder a la interrogante planteada en el segundo objetivo específico de la investigación.

Desde que tienen consciencia, los/as jóvenes están expuestas a experiencias de violencia de género, las que desde lo simbólico y metasimbólico, influyen y hasta determinan sus identidades y, por tanto, sus experiencias de vida. De este modo la violencia contra la mujer sería un fenómeno que, aunque consecuencia del sistema cultural, se vuelve inherente a las individualidades de todas las personas (Marañón, 2018), ratificando una realidad naturalizada y totalizadora (Bourdieu, 2000; Varela, 2017; Sendón de León, 2003), que contamina cada una de las células y estructuras sociales. 
Parte de dicha violencia continúa volcándose en la rigidez de roles y estereotipos de género, que se mantienen en la tradición machista (Colás y Villaciervos, 2007) y dificultan la apertura a paradigmas más diversos, flexibles y empáticos. Los desempeños sociales de hombres reflejan la misma asimetría de antaño e, inclusive, la aumentan, al imponer sobre las figuras femeninas nuevos roles sin transformar los habituales (Lagarde, 2004).

Como el sistema patriarcal de antaño continúa moldeando las identidades, los procesos de endoculturación (Rocha, 2009) y aprendizaje social (Bandura, 1977; Bandura y Walters, 1963) no hacen más que explicar cómo los/as jóvenes interiorizan - especialmente durante la niñez- patrones machistas provenientes de madres, profesores (de género masculino) y pareja. Aprenden a ser hombres y mujeres gracias a la (re)producción de construcciones de género normalizadas (Butler, 1997; Coll-Planas, 2013, de Beauvoir, 1999; Segato, 2016; López, 2016) y de un sinnúmero de (hetero)normas que definen su entorno y que les permiten sentirse parte de él.

Las redes sociales, los espacios de amistad, familia y la escuela formal resultaron ser escenarios recurrentes en la experimentación de violencia basada en género. En el caso de las plataformas virtuales, los malos tratos simbólicos están asociados a la sobre exposición por búsqueda de aprobación, práctica que deriva en conductas de hipersexualización y cosificación femenina (Marañón, 2018; Varela, 2017; Tortajada, 2018).

A este respecto, el cuerpo y la autoimagen destacan como elementos especialmente importantes para las jóvenes, quienes ven concentradas sus expectativas de desarrollo personal en la medida que son capaces de alcanzar parámetros de belleza, erotización y fertilidad (de Beauvoir, 1999; Marañón, 2018; López, 2016). Como consecuencia, surgen frecuentes sentimientos de inconformidad y frustración, toda vez que ser mujer se convierte en una búsqueda constante de perfección.

La juventud se presenta como una condición de continua transformación (Duarte, 2018), pero que -contextualizada en occidente- obedece a lógicas eminentemente hegemónicomasculinas. Sin embargo, en el discurso de los/as jóvenes (desde sus facetas políticotransformadoras) surgen ciertos senderos conducentes a la evolución y equidad, como la necesidad de sistemas escolares coeducativos, de contenidos audiovisuales que -explícita e implícitamente- versen sobre diversidad, empatía e inclusión, y de instancias de diálogo crítico que fomenten nuevas maneras de comprender y vivenciar el género, lejos del binarismo que hasta ahora nos ha adoctrinado.

Lograr el despertar cognitivo frente al machismo y la violencia que acarrea consigo implica una tarea titánica, pero no imposible. Es precisamente a través de la visibilización de elementos, acciones y actitudes cotidianas que podemos develar la artificialidad de la realidad simulada (Varela, 2017) en la que nos desenvolvemos y hacer frente a las injusticias de un sistema patriarcal que perjudica las identidades y experiencias de todos/as quienes forman 
Revista Punto Género N ․ 16 Diciembre de 2021

ISSN 2735-7473 / 79- 101

parte y que, con -demasiada- frecuencia, cobra la vida de niñas y mujeres en todas las esferas sociales. Toca tirar del velo y delatar, visibilizar las inequidades disfrazadas de normalidad.

\section{Referencias bibliográficas}

Acevedo, D., Biaggii, Y. y Borges, G. (2009): Violencia de género en el trabajo: acoso sexual y hostigamiento laboral. Revista venezolana de estudios de la mujer, 14(32), p.163182. $\quad$ http://ve.scielo.org/scielo.php?script=sci_arttext\&pid=S1316$37012009000100012 \& \operatorname{lng}=$ es\&tlng=es.

Aguilera Ruiz, O. (2009): Los estudios sobre juventud en Chile: coordenadas para un estado del arte. Ultima década, $17(31), \quad$ p.109-127. https://scielo.conicyt.cl/scielo.php?script=sci arttextypid=S0718$\underline{22362009000200007}$

Alegría del Ángel, M. y Rodríguez Barraza, A. (2015): Violencia en el noviazgo: perpetración, victimización y violencia mutua. Una revisión. Actualidades en Psicología. 29(118) p.57-72. https://www.redalyc.org/pdf/1332/133239321007.pdf

Alpízar, L. y Bernal, M. (2013): La Construcción Social de las Juventudes. Última década, 11(19), p.105-123. https://dx.doi.org/10.4067/S0718-22362003000200008

Arancibia Garrido, J., Billi, M., y Guerrero González, M. (2017): ¡Tu 'piropo' me violenta! Hacia una definición de acoso sexual callejero como forma de violencia de género. Revista Punto Género, (7),

6270

p.112-137. https://revistas.uchile.cl/index.php/RPG/article/view/46270

Axt, J. C. P., Lagos, L. A., y Henríquez, V. B. (2019): Los celos como norma emocional en las dinámicas de violencia de género en redes sociales en las relaciones de pareja de estudiantes de Temuco, Chile. Multidisciplinary Journal of Gender Studies, 8(2), p.180-203. https://hipatiapress.com/hpjournals/index.php/generos/article/view/4223

Bandura, A. (1977): Social Learning Theory. General Learning Press. https://scholar.google.com/scholar lookup?title=Social\%20learning\%20theory\&autho r=A.\%20Bandura\&publication year $=1977$

Bandura, A. y Walters, R. (1963): Aprendizaje social y desarrollo de la personalidad. Traducido del inglés. Alianza Editorial.

Bernal, R. F. (2005): Violencia de género en la escuela: sus efectos en la identidad, en la autoestima y en el proyecto de vida. Revista Iberoamericana de educación, (38), 6786. https://rieoei.org/RIE/article/view/831 
Revista Punto Género N․o 16 Diciembre de 2021

ISSN 2735-7473 / 79- 101

Bodelón, E. (2014): Violencia institucional y violencia de género. In Anales de la cátedra Francisco Suárez

p.131-155.

http://revistaseug.ugr.es/index.php/acfs/article/download/2783/2900

Bonilla-García, M y López-Suárez, A. (2016): Ejemplificación del proceso metodológico de la teoría fundamentada.

https://scielo.conicyt.cl/scielo.php?script=sci abstractypid=S0717$\underline{554 X 2016000300006 y / n g=e s y n r m=i s o}$

Espinar Ruiz, E., Mateo Pérez, M. A. (2020) Violencia de género: reflexiones conceptuales, derivaciones prácticas. Papers: revista de sociologia, , 86, p. 189-01. https://papers.uab.cat/article/view/v86-espinar-mateo

Butler, J. (2007): El género en disputa. El feminismo y la subversión de la identidad. Paidós.

Colás Bravo, P., y Villaciervos Moreno, P. (2007): La interiorización de los estereotipos de género en jóvenes y adolescentes. Revista De Investigación Educativa, 25(1), 35-38. https://revistas.um.es/rie/article/view/96421

Coll-Planas, G. (2013): Dibujando el género. EGALES.

De Beauvoir, S. (1999): El segundo sexo. Editorial Sudamericana.

De Miguel, A. (2005): El movimiento feminista y la redefinición de la realidad. Conferencia impartida en el Consejo de las Mujeres del Municipio de Madrid. http://www.derechoshumanos.unlp.edu.ar/assets/files/documentos/movimientofeminista-y-redefinicion-de-la-realidad.pdf

Díaz Berr, X., Mauro Cardarelli, A., Ansoleaga Moreno, E. y Toro Cifuentes, J. P. (2017): Violencia de género en el trabajo en Chile. Un campo de estudio ignorado. Ciencia y trabajo, 19(58),

p.42-48, https://scielo.conicyt.cl/scielo.php?script=sci abstractypid=S0718$24492017000100042 y \mid n g=e s y n r m=i$

Donoso Vázquez, T., Rubio Hurtado, M.J., y Vilà Baño, R. (2018): La adolescencia ante la violencia de género 2.0: Concepciones, conductas y experiencias. Educación XX1, 21(1), 109-134. doi: 10.5944/educXX1.20180

Duarte Quapper, K. (2018): Investigación social chilena en juventudes. El caso de la revista Última Década. Ultima década, 26(50), 124-154. https://dx.doi.org/10.4067/S0718$\underline{22362018000300124}$ 
Revista Punto Género N․o 16 Diciembre de 2021

ISSN 2735-7473 / 79- 101

Espinar Ruiz, E. y Mateo Pérez, M. (2020): Violencia de género: reflexiones conceptuales, derivaciones prácticas. Papers. Revista de Sociología. [S.I.], 86, 189-201. https://papers.uab.cat/article/view/v86-espinar-mateo/pdf-es

Facio, A. Y Fries, L. (20051999): Feminismo, género y patriarcado. Academia. Revista sobre Enseñanza del Derecho de Buenos Aires, 3(6). http://repositorio.ciem.ucr.ac.cr/ispui/handle/123456789/122

Fontenla, M. (2008): ¿Que es el patriarcado? Diccionario de estudios de género y feminismo, Editorial Biblos. https://www.mujeresenred.net/spip.php?article1396

Gómez, J.; Latorre A.; Sánchez, M. y Flecha, R. (2016): Metodología Comunicativa Crítica. El Roure editorial.

Gómez, M. C. S., Vicario, B. P. y García, A. V. M. (2015): Indicadores de violencia de género en las relaciones amorosas. Estudio de caso en adolescentes chilenos. Pedagogía social: revista interuniversitaria, (26), p.85-109. https://dialnet.unirioja.es/servlet/articulo?codigo $=5099212$

González, A. L. R., y Carapia, J. D. C. A. C. (2015): Percepción de la violencia de género en las y los jóvenes universitarios. Centro de Estudios de la Mujer, ENTS-UNAM, https://eventos.ucol.mx/content/micrositios/241/file/memoria/pdf/50.pdf

Hernández, R., Fernández, C. y Baptista, P. (2003): Metodología de la investigación (3ª ed.). Editorial Mc Graw-Hill.

Lagarde, M. (2004): Mujeres cuidadoras: entre la obligación y la satisfacción. En Cuidar cuesta: Costes y beneficios del cuidado, Congreso Internacional SARE 2003: 155-160. Vitoria-Gasteiz: Emakunde, Instituto Vasco de la Mujer. http://pmayobre.webs.uvigo.es/textos/marcela lagarde y de los rios/mujeres cuida doras entre la obligacion y la satisfaccion lagarde.pdf

López Aguilera, I. (2016): Sexismo y roles de género en jóvenes. Y su relación con los contextos de socialización (Trabajo de fin de Máster, Universidad Pontificia Comillas). https://repositorio.comillas.edu/xmlui/handle/11531/13386

Lorente, M. (2009): Los nuevos hombres nuevos. Los miedos de siempre en tiempos de igualdad. Destino. 
Revista Punto Género N ․ 16 Diciembre de 2021

ISSN 2735-7473 / 79- 101

Madrid, S. (2006): Profesorado, política educativa y género en Chile. Balance y propuestas. Colección Ideas, 8(76). https://www.researchgate.net/publication/237795181 Profesorado politica educativa y genero en Chile Balance y propuestas

Marañón, I. (2018): Educar en el feminismo. Plataforma Editorial.

Martín, V. R. (2012): Adolescentes y jóvenes de Castilla La Mancha ante la violencia de género en las relaciones de pareja (Doctoral dissertation, Universidad de Castilla-La Mancha). https://dialnet.unirioja.es/servlet/tesis?codigo=124295

Parsons, T. (1968): The position of identity in the General Theory of Action. In C. Gordon \& K. Gergen (Eds.), The self in social interaction, p. 16-23. New York: Wiley.

Pereira Pérez, Z. (2011): Los diseños de método mixto en la investigación en educación: Una experiencia concreta. Revista Electrónica Educare, XV(1), p.15-29. https://www.redalyc.org/articulo.oa?id=1941/194118804003

Ramírez Rodríguez, J. C., López López, G. C. y Padilla González, F. J. (2009): ¿Nuevas generaciones, nuevas creencias? Violencia de género y jóvenes. La ventana. Revista de estudios de género, 3(29), p.110-145. http://www.scielo.org.mx/scielo.php?script=sci arttextypid=S1405$\underline{94362009000100006 y|n g=e s y t| n g=e s}$.

Rocha, T. (2009): Desarrollo de la identidad de género desde una perspectiva psico-sociocultural: un recorrido conceptual. Periódicos electrónicos en Psicología, 43, (2), p. 250-259.

https://www.researchgate.net/publication/45492428 Desarrollo de la Identidad de Genero desde una Perspectiva Psico-Socio-Cultural Un Recorrido Conceptual

Rodríguez-Castro, Y., y Alonso-Ruido, P. (2015): Análisis de los discursos de los y las jóvenes sobre la violencia en las relaciones de pareja. Revista de Estudios e Investigación en Psicología y Educación, 015-018. https://revistas.udc.es/index.php/reipe/article/view/reipe.2015.0.02.235

Ruiz, V.R., Carbonell, X., y Oberst, U. (2012): Redes sociales on-line, género y construcción del self. Aloma. Revista de Psicologia, Ciències de l'Educació i de l'Esport, 30(2), p. 97107, http://www.revistaaloma.net/index.php/aloma/article/view/166 
Revista Punto Género N․o 16 Diciembre de 2021

ISSN 2735-7473 / 79- 101

Ruiz-Pérez, I. y Pastor-Moreno, G. (2020): Medidas de contención de la violencia de género durante la pandemia de COVID-19, Gaceta Sanitaria, 35(4), p. 389-394. https://doi.org/10.1016/i.gaceta.2020.04.005

San Martín Cantero, D.. (2014): Teoría fundamentada y Atlas.ti: recursos metodológicos para la investigación educativa REDIE. Revista Electrónica de Investigación Educativa, 16(1), p. 104-122. https://www.redalyc.org/pdf/155/15530561008.pdf

Segato, L. (2016): La guerra contra las mujeres. Traficantes de Sueños

Sendón de León, V. (2003): Mujeres en la Era Global. Contra un patriarcado neoliberal. Icaria Editorial S.A.

Sendón de León, V. (30 de mayo de 2019): Victoria Sendón de León: Si no cuestionas el modelo, la igualdad sirve para muy poco. Fundación Woman's week. https://madridwomans-week.com/victoria-sendon-de-leon-si-no-cuestionas-el-modelo-la-igualdadsirve-para-muy-pocol

Strauss, A. L. y Corbin, J. (2002): Bases de la investigación cualitativa: técnicas y procedimientos para desarrollar la teoría fundada (1. ed.). Editorial Universidad de Antioquia.

Tortajada, I.; Willem; C. y Araüna, N. (2018): Género y redes sociales: retos para la construcción de la identidad adolescente. En Ed. Jiménez, S.; Garmendia, M. y Casado, M.A. Entre selfies $y$ whatsapps. Oportunidades y riesgos para la infancia y la adolescencia conectada. 1era ed., Vol. 01, p.91-103. Gedisa.

Varela, N. (2017): Cansadas. Ediciones B S.A.

Zamudio Sánchez, F. J., Andrade Barrera, M. A., Arana Ovalle, R. I. y Alvarado Segura, A. A. (2017): Violencia de género sobre estudiantes universitarios (as). Convergencia, 24(75),

p.133-157. http://www.scielo.org.mx/scielo.php?script=sci abstractypid=S1405$14352017000300133 \mathrm{ylng}=$ esynrm=iso

Zapata-Sepúlveda, P., Fernández-Dávila, P., y Sánchez-Gómez, M. C. (2012): Violencia de género en mujeres con ascendencia étnica aymara en el extremo norte de Chile. Revista de Psiquiatría y Salud Mental, 5(3), p.167-172. https://medes.com/publication/84512 
Revista Punto Género N․ำ 16 Diciembre de 2021

ISSN 2735-7473 / 79- 101

Zurbano Berenguer, B., Liberia Vayá, I. y Barredo Ibáñez, D. (2015). El concepto social de la violencia contra las mujeres en España: aproximaciones a los imaginarios de jóvenes estudiantes. Revista interdisciplinaria de estudios de género de El Colegio de México, $1(2)$, 145-169.

http://www.scielo.org.mx/scielo.php?script=sci arttext\&pid=S2395-

91852015000200145\&lng=es\&tlng=es. 